\title{
Some result's in the theory of fractional integral equations of Volterra-Fridlhom types
}

\author{
Raad N. Butris ${ }^{1}$, Faraj Y.Ishak ${ }^{2}$ \\ ${ }^{1}$ Department of Mathematics, Collage of Basic Education, Duhok University, Kurdistan region, Iraq \\ raad.butris@uod.ac \\ ${ }^{2}$ Department of Statistics, Collage of Adminstration \& Economics, Duhok University, Kurdistan region, Iraq \\ faraj.ishak@uod.ac
}

\begin{abstract}
The aim of this work is finding some result's in the existence, uniqueness and stability solutions of new fractional integral equations of Volterra Fridlhom types by using Picard approximation method. Theorems on existence and uniqueness of a solution are established under some necessary and sufficient conditions on compact spaces. Furthermore, the study leads us to improve and extend the above method and the study become more general and detailed than those introduced by Butris results.
\end{abstract}

Key ords: Existence, uniqueness, and stability solution, fractional integral equations of Volterra-Fridlhom types, Picard approximation method.

\section{INTRODUCTION}

Over the years, many mathematicians, using their own notation and approach, have found various definitions that fit the idea of a non-integer order integral or derivative. One version that has been popularized in the world of fractional calculus is the Riemann Liouville definition. It is interesting to note that the Riemann-Liouville definition of a fractional derivative gives the same result. Since most of the other definitions of fractional calculus are largely variations of the Riemann-Liuoville version.

Many results about the existence, uniqueness and stability solutions of fractional integral equations have been obtain by Picard approximation method that were proposed by many studies $[2,3,5,7,9,18,19,20]$. They are many subjects in physics and technology using mathematical methods that depends on the linear and nonlinear integral equations, and it became clear that the existence of solutions and it is algorithm structure from more important problems in many studies and researches $[4,6,10,11,17,18]$ dedicates for treatment the autonomous and non-autonomous systems and specially with integral equations. Picard approximation method [11,12] owing to the great possibilities of exploiting computers are becoming versatile means of the finding and approximate construction of a solution of integral equations. Rama [17 ]assumes the Picard approximation method to study the ac solutions for integral equations and it is algorithm structure and this method include uniformly sequences of a functions and the results of that study is using of the a solutions on wide range in the difference of new processes industry and technology on the studies[1,2,3,8,13,14,15,16,21,22].

Definition1[17]. Let $\left\{f_{m}(t)\right\}_{m=0}^{\infty}$ be a sequence of functions defined on a set. $E \subseteq R^{1}$ We say that $\left\{f_{m}(t)\right\}_{m=0}^{\infty}$ converges uniformly to the limit function $f$ on $E$ if, given $\varepsilon>0$ there exists a positive integer $N$ such that :

$$
\left|f_{m}(t)-f(t)\right|<\varepsilon, \quad(m \geq N, t \in E
$$

Definition2[17 ]: Let $\mathrm{f}$ be a continuous function defined on a domain

$$
\mathrm{D}=\{(\mathrm{t}, \mathrm{u}): \mathrm{a} \leq \mathrm{t} \leq \mathrm{b}, \mathrm{c} \leq \mathrm{u} \leq \mathrm{d}\} .
$$

Then $\mathrm{f}$ is said to satisfy a Lipchitz condition in the variable $\mathrm{u}$ on $\mathrm{D}$, provided that a constant $\mathrm{L}>0$ exists with property that $\left|\mathrm{f}\left(\mathrm{t}, \mathrm{u}_{1}\right)-\mathrm{f}\left(\mathrm{t}, \mathrm{u}_{2}\right)\right| \leq \mathrm{L} \mathrm{u}_{1}-\mathrm{u}_{2} \mid$, for all $\left(\mathrm{t}, \mathrm{u}_{1}\right),\left(\mathrm{t}, \mathrm{u}_{2}\right) \in \mathrm{D}$. the constant $\mathrm{L}$ is called a Lipchitz constant for $\mathrm{f}$.

Definition3 [17].A solution $u(t)$ is said to be stable if for each $\varepsilon>0$, there exists a $\delta>0$ such that any solution $\breve{\mathrm{u}}(\mathrm{t})$ which satisfies $\left\|\breve{\mathrm{u}}\left(\mathrm{t}_{0}\right)-\mathrm{u}\left(\mathrm{t}_{0}\right)\right\|<\delta$ for some $\mathrm{t}_{0}$, also satisfies $\|\breve{\mathrm{u}}(\mathrm{t})-\mathrm{u}(\mathrm{t})\|<\varepsilon$ for all $\mathrm{t} \geq \mathrm{t}_{0}$.

Definition 4[9]. If $g(t) \epsilon[a, b]$ and, then the operator $I_{a+}^{\alpha}$ defined by

$$
I_{a+}^{\alpha} g(x)=\frac{1}{\Gamma(\alpha)} \int_{a}^{t} \frac{g(s)}{(t-s)^{1-\alpha}} d s .
$$

for $a \leq t \leq b$, is called the Riemann-Liouville fractional integral operator of order $\alpha$. Here $\Gamma(\alpha)$ is the Gamma function defined by:

$$
\Gamma(x)=\int_{0}^{\infty} t^{x-1} e^{-t} d t .
$$

Definition 5[9]. The Caputo fractional derivative of order $\alpha \geq 0$ of a continuous function 
$\mathrm{g}:(\mathrm{a}, \mathrm{b}) \longrightarrow$ Bis defined by

$$
{ }^{c} D_{a+}^{\alpha} g(t)=\frac{1}{\Gamma(n-\alpha)} \int_{a}^{t} \frac{g^{n}(s)}{(t-s)^{\alpha-n-1}} d s .
$$

Butris [1] used both methods Picard approximation and Banach fixed point theorems for studying the existence and uniqueness solutions to the following Volterra integral equations: -

$$
\mathrm{u}(\mathrm{t})=\mathrm{f}(\mathrm{t})+\int_{\mathrm{a}}^{\mathrm{t}} \mathrm{F}(\mathrm{t}, \mathrm{s}) \mathrm{u}(\mathrm{s}) \mathrm{ds}, \quad(\mathrm{t} \in[0, \mathrm{a}])
$$

In this equation the functions $\mathrm{f}(\mathrm{t})$ and $\mathrm{F}(\mathrm{t}, \mathrm{s})$ be continuous on (finite) interval $0 \leq \mathrm{t} \leq \mathrm{a}$ and the square region $0 \leq \mathrm{t} \leq \mathrm{a}$, $\mathrm{o} \leq \mathrm{s} \leq \mathrm{a}$, respectively.

Our work is extending some results of Butris [1], by using the Picard approximation method only.

Consider the following fractional integrals equations of Volterra-Fridlhom types: -

$$
\mathrm{u}(\mathrm{t})=\mathrm{f}(\mathrm{t})+\frac{1}{\Gamma(\alpha)} \int_{\mathrm{a}}^{\mathrm{t}}(\mathrm{t}-\mathrm{s})^{\alpha-1} \mathrm{~F}(\mathrm{t}, \mathrm{s}, \mathrm{u}(\mathrm{s}), \mathrm{w}(\mathrm{s})) \mathrm{ds}
$$

and

$$
w(t)=g(t)+\frac{1}{\Gamma(\alpha)} \int_{a}^{b}(t-s)^{\alpha-1} G(t, s, u(s), w(s)) d s
$$

where

$\mathrm{u} \in \mathrm{D}_{1} \subseteq \mathrm{R}^{\mathrm{n}}, \mathrm{w} \in \mathrm{D}_{2} \subseteq \mathrm{R}^{\mathrm{n}}, \mathrm{D}_{1}, \mathrm{D}_{2}$ are close and bounded domains.

The vector functions $\mathrm{f}(\mathrm{t}), \mathrm{g}(\mathrm{t})$ and $\mathrm{F}(\mathrm{t}, \mathrm{u}, \mathrm{w}), \mathrm{G}(\mathrm{t}, \mathrm{u}, \mathrm{w})$ are define and continuous on the domain:-

$\mathrm{D}=$

$\left\{(\mathrm{t}, \mathrm{s}, \mathrm{u}, \mathrm{w}) ; \mathrm{t}, \mathrm{s} \in \mathrm{R}^{1},|t-s| \leq h, \mathrm{u} \in \mathrm{D}_{1}, \mathrm{w} \in\right.$

$$
\left.\mathrm{D}_{2}, 0<\propto<1\right\}
$$

Suppose that the vector functions $\mathrm{F}(\mathrm{t}, \mathrm{u}, \mathrm{w})$ and $\mathrm{G}(\mathrm{t}, \mathrm{u}, \mathrm{w})$ satisfy the following inequalities

$$
\begin{aligned}
& \|\mathrm{F}(\mathrm{t}, \mathrm{s}, \mathrm{u}, \mathrm{w})\| \leq \mathrm{M}_{\alpha}, \quad\|\mathrm{G}(\mathrm{t}, \mathrm{s}, \mathrm{u}, \mathrm{w})\| \leq \mathrm{N}_{\alpha} \\
& \begin{aligned}
\| \mathrm{F}\left(\mathrm{t}, \mathrm{s}, \mathrm{u}_{1}, \mathrm{w}_{1}\right)- & \mathrm{F}\left(\mathrm{t}, \mathrm{s}, \mathrm{u}_{2}, \mathrm{w}_{2}\right) \| \\
& \leq \mathrm{K}_{\alpha}\left(\left\|\mathrm{u}_{1}-\mathrm{u}_{2}\right\|+\left\|\mathrm{w}_{1}-\mathrm{w}_{2}\right\|\right) \\
\| \mathrm{G}\left(\mathrm{t}, \mathrm{s}, \mathrm{u}_{1}, \mathrm{w}_{1}\right)- & \mathrm{G}\left(\mathrm{t}, \mathrm{s}, \mathrm{u}_{2}, \mathrm{w}_{2}\right) \| \\
& \leq \mathrm{L}_{\alpha}\left(\left\|\mathrm{u}_{1}-\mathrm{u}_{2}\right\|+\left\|\mathrm{w}_{1}-\mathrm{w}_{2}\right\|\right)
\end{aligned}
\end{aligned}
$$

For all $\mathrm{t}, \mathrm{s} \in \mathrm{R}^{1} \mathrm{u}, \mathrm{u}_{1}, \mathrm{u}_{2} \in \mathrm{D}_{1}, \mathrm{w}, \mathrm{w}_{1}, \mathrm{w}_{2} \in \mathrm{D}_{2}$

where $\mathrm{M}_{\alpha}, \mathrm{N}_{\alpha}, \mathrm{K}_{\alpha}$ and $\mathrm{L}_{\alpha}$ are positive constant and

$$
\|.\|=\max _{\mathrm{t} \in[0, \mathrm{~h}]}|.| \quad \text { (3) . }
$$

We define non-empty sets as follows: -

$$
\left.\begin{array}{l}
\mathrm{D}_{\mathrm{F}}=\mathrm{D}_{1}-\frac{\mathrm{h}^{\alpha} \mathrm{M}_{\alpha}}{\alpha \Gamma(\alpha)}+q_{1}+\|\mathrm{f}(\mathrm{a})\| \\
\mathrm{D}_{\mathrm{G}}=\mathrm{D}_{2}-\frac{\mathrm{h}^{\alpha} \mathrm{N}_{\alpha}}{\alpha \Gamma(\alpha)}+q_{2}+\|\mathrm{g}(\mathrm{a})\|
\end{array}\right\}
$$

Where $q_{1}=\max _{\mathrm{t} \in[0, \mathrm{~h}]}|f(t)|$ and $q_{2}=\max _{\mathrm{t} \in[0, \mathrm{~h}]}|g(t)|$ Furthermore, we assume that the largest Eigen-value of the matrix

$$
\omega=\left(\begin{array}{cc}
\mathrm{K}_{\alpha} \frac{\mathrm{h}^{\alpha}}{\alpha \Gamma(\alpha)} & \mathrm{K}_{\alpha} \frac{\mathrm{h}^{\alpha}}{\alpha \Gamma(\alpha)} \\
\mathrm{L}_{\alpha} \frac{\mathrm{h}^{\alpha}}{\alpha \Gamma(\alpha)} & \mathrm{L}_{\alpha} \frac{\mathrm{h}^{\alpha}}{\alpha \Gamma(\alpha)}
\end{array}\right)
$$

less than one, i.e.

$$
\varphi_{\max }(\omega)=\frac{\mathrm{h}^{\alpha}}{\alpha \Gamma(\alpha)}\left(\mathrm{K}_{\alpha}+\mathrm{L}_{\alpha}\right)<1
$$

Define a sequence of functions $\left\{\mathrm{u}_{\mathrm{m}}(\mathrm{t})\right\}_{\mathrm{m}=0}^{\infty}$ and $\left\{\mathrm{w}_{\mathrm{m}}(\mathrm{t})\right\}_{\mathrm{m}=0}^{\infty}$ by the following: -

$\mathrm{u}_{\mathrm{m}}(\mathrm{t})=\mathrm{f}(\mathrm{t})+\frac{1}{\Gamma(\alpha)} \int_{\mathrm{a}}^{\mathrm{t}}(\mathrm{t}-\mathrm{s})^{\alpha-1} \mathrm{~F}\left(\mathrm{t}, \mathrm{s}, \mathrm{u}_{\mathrm{m}-1}(\mathrm{~s}), \mathrm{w}_{\mathrm{m}-1}(\mathrm{~s})\right) \mathrm{ds}$

With $\quad \mathrm{u}_{0}(\mathrm{a})=\mathrm{f}(\mathrm{a})$

and

$\mathrm{w}_{\mathrm{m}}(\mathrm{t})=\mathrm{g}(\mathrm{t})+\frac{1}{\Gamma(\alpha)} \int_{\mathrm{a}}^{\mathrm{b}}(\mathrm{t}-\mathrm{s})^{\alpha-1} \mathrm{H}\left(\mathrm{t}, \mathrm{s}, \mathrm{u}_{\mathrm{m}-1}(\mathrm{~s}), \mathrm{w}_{\mathrm{m}-1}(\mathrm{~s})\right) \mathrm{ds}$

With $\quad \mathrm{w}_{0}(\mathrm{a})=\mathrm{g}(\mathrm{a})$

\section{EXISTENCE SOLUTIONS OF FRACTIONAL INTEGRAL EQUATIONS OF (V) AND(F).}

The investigation of the existence solution of $(\mathrm{V})$ and $(\mathrm{F})$ will be the introduced by the following theorem: -

Theorem1. Let the vector functions $f(t), g(t)$ and $\mathrm{F}(\mathrm{t}, \mathrm{u}, \mathrm{w}), \mathrm{G}(\mathrm{t}, \mathrm{u}, \mathrm{w})$ defined and continuous on the domain (1) suppose these functions are satisfying the inequalities (2), (3) and the conditions (4), (5). Then there exists a sequence of functions (6) and (7) converges uniformly on the domain

$$
D^{*}=\left\{((t, s), f(a), g(a)) \in[0, h] \times D_{1} \times D_{2}\right\}
$$

to the limit vector function $\left(\begin{array}{c}u(t) \\ w(t)\end{array}\right)$ which is a continuous on the domain (1) and satisfies the following integral equations: -

$\left(\begin{array}{c}\mathrm{u}(\mathrm{t}) \\ \mathrm{w}(\mathrm{t})\end{array}\right)=$

$\left(\begin{array}{l}\mathrm{f}(\mathrm{t})+\frac{1}{\Gamma(\alpha)} \int_{\mathrm{a}}^{\mathrm{t}}(\mathrm{t}-\mathrm{s})^{\alpha-1} \mathrm{~F}\left(\mathrm{t}, \mathrm{s}, \mathrm{u}_{\mathrm{m}-1}(\mathrm{~s}), \mathrm{w}_{\mathrm{m}-1}(\mathrm{~s})\right) \mathrm{ds} \\ \mathrm{g}(\mathrm{t})+\frac{1}{\Gamma(\alpha)} \int_{\mathrm{a}}^{\mathrm{b}}(\mathrm{t}-\mathrm{s})^{\alpha-1} \mathrm{H}\left(\mathrm{t}, \mathrm{s}, \mathrm{u}_{\mathrm{m}-1}(\mathrm{~s}), \mathrm{w}_{\mathrm{m}-1}(\mathrm{~s})\right) \mathrm{ds}\end{array}\right)$

and it is existing solution of $(\mathrm{V})$ and $(\mathrm{F})$,pprovide that:

$$
\left(\begin{array}{c}
\left\|\mathrm{u}_{\mathrm{m}}(\mathrm{t})-\mathrm{f}(\mathrm{a})\right\| \\
\left\|\mathrm{w}_{\mathrm{m}}(\mathrm{t})-\mathrm{g}(\mathrm{a})\right\|
\end{array}\right) \leq\left(\begin{array}{c}
\frac{\mathrm{h}^{\alpha} \mathrm{M}_{\alpha}}{\alpha \Gamma(\alpha)}+q_{1}+\|\mathrm{f}(\mathrm{a})\| \\
\frac{\mathrm{h}^{\alpha} \mathrm{N}_{\alpha}}{\alpha \Gamma(\alpha)}+q_{2}+\|\mathrm{g}(\mathrm{a})\|
\end{array}\right)
$$

and

$$
\left(\begin{array}{c}
\left\|u_{m+1}(t)-f(a)\right\| \\
\left\|w_{m+1}(t)-g(a)\right\|
\end{array}\right) \leq \omega^{m}(E-\omega)^{-1} \emptyset_{0}
$$

for all $\mathrm{t} \in[0, \mathrm{~h}]$ and $\mathrm{f}(\mathrm{a}) \in \mathrm{D}_{\mathrm{F}}, \mathrm{g}(\mathrm{a}) \in \mathrm{D}_{\mathrm{G}}, \mathrm{m}=0,1, \ldots$ where:

$$
\emptyset_{0}=\left(\begin{array}{l}
\frac{\mathrm{h}^{\alpha} \mathrm{M}_{\alpha}}{\alpha \Gamma(\alpha)}+q_{1}+\|\mathrm{f}(\mathrm{a})\| \\
\frac{\mathrm{h}^{\alpha} \mathrm{N}_{\alpha}}{\alpha \Gamma(\alpha)}+q_{2}+\|\mathrm{g}(\mathrm{a})\|
\end{array}\right)
$$

By mathematical indication we can prove that:

$$
\left\|\mathrm{u}_{\mathrm{m}}(\mathrm{t})-\mathrm{u}_{0}\right\| \leq \frac{\mathrm{h}^{\alpha} \mathrm{M}_{\alpha}}{\alpha \Gamma(\alpha)}+q_{1}+\|\mathrm{f}(\mathrm{a})\|
$$

That is $u_{m}(t) \in D_{F}$, for all $t \in[0, h]$ and $f(a) \in D_{F}$ 
Raad N. Butris et al., International Journal of Information Systems and Computer Sciences, 9(1), January - February 2020,5 -10

Similarly, from the sequence of functions (7) and we obtain that

$$
\left\|\mathrm{w}_{\mathrm{m}}(\mathrm{t})-\mathrm{g}(\mathrm{a})\right\| \leq \frac{\mathrm{h}^{\alpha} \mathrm{N}_{\alpha}}{\alpha \Gamma(\alpha)}+q_{2}+\|\mathrm{g}(\mathrm{a})\|
$$

that is $\mathrm{w}_{\mathrm{m}}(\mathrm{t}) \in \mathrm{D}_{\mathrm{G}}$, for all $\mathrm{t} \in[0, \mathrm{~h}]$ and $\mathrm{g}(\mathrm{a}) \in \mathrm{D}_{\mathrm{G}}$

Next, we shall prove that the sequences of functions (6) and (7) converge uniformly on the domain (8). Then by mathematics induction, we have

$$
\begin{array}{r}
\left\|\mathrm{u}_{\mathrm{m}+1}(\mathrm{t})-\mathrm{u}_{\mathrm{m}}(\mathrm{t})\right\| \leq \mathrm{K}_{\alpha} \frac{\mathrm{h}^{\alpha}}{\alpha \Gamma(\alpha)} \quad\left(\left\|\mathrm{u}_{\mathrm{m}}(\mathrm{t})-\mathrm{u}_{\mathrm{m}-1}(\mathrm{t})\right\|+\right. \\
\left.\left\|\mathrm{w}_{\mathrm{m}}(\mathrm{t})-\mathrm{w}_{\mathrm{m}-1}(\mathrm{t})\right\|\right)
\end{array}
$$

and

$$
\begin{array}{r}
\left\|\mathrm{w}_{\mathrm{m}+1}(\mathrm{t})-\mathrm{w}_{\mathrm{m}}(\mathrm{t})\right\| \leq \mathrm{L}_{\alpha} \frac{\mathrm{h}^{\alpha}}{\alpha \Gamma(\alpha)}\left(\left\|\mathrm{u}_{\mathrm{m}}(\mathrm{t})-\mathrm{u}_{\mathrm{m}-1}(\mathrm{t})\right\|+\right. \\
\left.\left\|\mathrm{w}_{\mathrm{m}}(\mathrm{t})-\mathrm{w}_{\mathrm{m}-1}(\mathrm{t})\right\|\right)
\end{array}
$$

for all $\mathrm{m}=1,2,3, \ldots$.

Rewrite (14) and (15) in a vector form we get:

where

$$
\emptyset_{\mathrm{m}+1} \leq \omega(\mathrm{t}) \emptyset_{\mathrm{m}}
$$

$\emptyset_{\mathrm{m}+1}=\left(\begin{array}{c}\left\|\mathrm{u}_{\mathrm{m}+1}(\mathrm{t})-\mathrm{u}_{\mathrm{m}}(\mathrm{t})\right\| \\ \left\|\mathrm{w}_{\mathrm{m}+1}(\mathrm{t})-\mathrm{w}_{\mathrm{m}}(\mathrm{t})\right\|\end{array}\right), \quad \emptyset_{\mathrm{m}}=\left(\begin{array}{c}\left\|\mathrm{u}_{\mathrm{m}}(\mathrm{t})-\mathrm{u}_{\mathrm{m}-1}(\mathrm{t})\right\| \\ \left\|\mathrm{w}_{\mathrm{m}}(\mathrm{t})-\mathrm{w}_{\mathrm{m}-1}(\mathrm{t})\right\|\end{array}\right)$ and

$\omega(\mathrm{t})=\left(\begin{array}{ll}\mathrm{K}_{\alpha} \frac{(\mathrm{t}-\mathrm{a})^{\alpha}}{\alpha \Gamma(\alpha)} & \mathrm{K}_{\alpha} \frac{(\mathrm{t}-\mathrm{a})^{\alpha}}{\alpha \Gamma(\alpha)} \\ \mathrm{L}_{\alpha} \frac{(\mathrm{t}-\mathrm{a})^{\alpha}}{\alpha \Gamma(\alpha)} & \mathrm{L}_{\alpha} \frac{(\mathrm{t}-\mathrm{a})^{\alpha}}{\alpha \Gamma(\alpha)}\end{array}\right)$

Now we, take the maximum value for the both sides of the inequality (16), we have

where $\omega=\max _{\mathrm{t} \in[0, \mathrm{~h}]} \omega(\mathrm{t})$.

$$
\emptyset_{\mathrm{m}+1} \leq \omega \emptyset_{\mathrm{m}}
$$

By repeating (17), we find that $\emptyset_{\mathrm{m}+1} \leq \omega^{\mathrm{m}} \emptyset_{0}$ and also, we get

$$
\sum_{i=1}^{m} \emptyset_{i} \leq \sum_{i=1}^{\infty} \omega^{i-1} \emptyset_{0}
$$

Using the condition (15), thus the sequence of functions (6) and (7) are uniformly convergent, that is

$$
\lim _{m \rightarrow \infty} \sum_{i=1}^{m} \omega^{i-1} \emptyset_{0} \leq \sum_{i=1}^{\infty} \omega^{i-1} \emptyset_{0}=(E-\omega)^{-1} \emptyset_{0}
$$

$$
\text { Let } \quad \lim _{m \rightarrow \infty}\left(\begin{array}{c}
u_{m}(t) \\
w_{m}(t)
\end{array}\right)=\left(\begin{array}{c}
u(t) \\
w(t)
\end{array}\right)
$$

Since the sequence of function (6) and (7) are defined and continuous in the domain (1), then the limit function $\left(\begin{array}{c}u(t) \\ w(t)\end{array}\right)$ is also defined and continuous in the domain (1).
By using the conditions and inequalities of a theorem, we can prove that the inequalities (10) and (11) will be satisfied for all $\mathrm{t}$ $\in[0, \mathrm{~h}], \mathrm{f}(\mathrm{a}) \in \mathrm{D}_{\mathrm{F}}, \mathrm{g}(\mathrm{a}) \in \mathrm{D}_{\mathrm{G}}, \quad \mathrm{m}=0,1,2 \ldots$

\section{UNIQUENESS SOLUTIONS OF FRACTIONAL INTEGRAL EQUATIONS OF (V) AND(F).}

The investigation of the uniqueness solutions of (V) and (F) will be introduced by:

Thorem2. Let all assumptions and conditions of Theorem 3 be satisfied. Then the solution $\left(\begin{array}{c}u(t) \\ w(t)\end{array}\right)$ is a unique of $(V)$ and $(F)$.

Proof. Let $\left(\begin{array}{c}\breve{u}(t) \\ \breve{w}(t)\end{array}\right)$ be another solution of $(V)$ and $(F)$, that is

$$
\breve{u}(t)=f(t)+\frac{1}{\Gamma(\alpha)} \int_{a}^{t}(t-s)^{\alpha-1} F(t, s, \breve{u}(s), \breve{w}(s)) d s
$$

and

$$
\breve{\mathrm{w}}(\mathrm{t})=\mathrm{g}(\mathrm{t})+\frac{1}{\Gamma(\alpha)} \int_{\mathrm{a}}^{\mathrm{b}}(\mathrm{t}-\mathrm{s})^{\alpha-1} \mathrm{G}(\mathrm{t}, \mathrm{s}, \breve{\mathrm{u}}(\mathrm{s}), \breve{\mathrm{w}}(\mathrm{s})) \mathrm{ds}
$$

Assuming

$$
\|\mathrm{u}(\mathrm{t})-\breve{\mathrm{u}}(\mathrm{t})\| \leq \mathrm{K}_{\alpha} \frac{\mathrm{h}^{\alpha}}{\alpha \Gamma(\alpha)}(\|\mathrm{u}(\mathrm{s})-\breve{\mathrm{u}}(\mathrm{t})\|+\| \mathrm{w}(\mathrm{s})-
$$$$
\breve{\mathrm{W}}(\mathrm{t}) \|)
$$

and

$$
\begin{gathered}
\|\mathrm{w}(\mathrm{t})-\breve{\mathrm{w}}(\mathrm{t})\| \leq \mathrm{L}_{\alpha} \frac{\mathrm{h}^{\alpha}}{\alpha \Gamma(\alpha)}\left(\|\mathrm{u}(\mathrm{t})-\breve{\mathrm{u}}(\mathrm{t})\|+\underset{\mathrm{w}(\mathrm{t}) \|)}{\| \mathrm{w}_{\mathrm{m}}(\mathrm{t})-}\right. \\
\left(\begin{array}{c}
\|\mathrm{u}(\mathrm{t})-\breve{\mathrm{u}}(\mathrm{t})\| \\
\|\mathrm{w}(\mathrm{t})-\breve{\mathrm{w}}(\mathrm{t})\|
\end{array}\right) \leq \omega\left(\begin{array}{c}
\|\mathrm{u}(\mathrm{t})-\breve{\mathrm{u}}(\mathrm{t})\| \\
\|\mathrm{w}(\mathrm{t})-\breve{\mathrm{w}}(\mathrm{t})\|
\end{array}\right)
\end{gathered}
$$

By iterating the inequality (21) we have:

$$
\left(\begin{array}{c}
\|\mathrm{u}(\mathrm{t})-\breve{\mathrm{u}}(\mathrm{t})\| \\
\|\mathrm{w}(\mathrm{t})-\breve{\mathrm{w}}(\mathrm{t})\|
\end{array}\right) \leq \omega^{\mathrm{m}}\left(\begin{array}{c}
\|\mathrm{u}(\mathrm{t})-\breve{\mathrm{u}}(\mathrm{t})\| \\
\|\mathrm{w}(\mathrm{t})-\breve{w}(\mathrm{t})\|
\end{array}\right)
$$

Then by the condition (15), we find that:

$$
\left(\begin{array}{c}
\|\mathrm{u}(\mathrm{t})-\breve{\mathrm{u}}(\mathrm{t})\| \\
\|\mathrm{w}(\mathrm{t})-\breve{\mathrm{w}}(\mathrm{t})\|
\end{array}\right) \rightarrow\left(\begin{array}{l}
0 \\
0
\end{array}\right)
$$

Thus $\left(\begin{array}{c}u(t) \\ w(t)\end{array}\right)=\left(\begin{array}{c}\breve{u}(t) \\ \check{w}(t)\end{array}\right)$. Hence the solutions $\left(\begin{array}{c}u(t) \\ w(t)\end{array}\right)$ of $\left(V_{1}\right)$ and $\left(\mathrm{V}_{2}\right)$ is a unique on the domain (1).

\section{4.. STABILITY SOLUTIONS OF FRACTIONAL INTEGRAL EQUATIONS OF (V) AND(F).}

In this section, we can study the stability solutions of Volterra integral equations $(\mathrm{V})$ and $(\mathrm{F})$ respectively.

Theorem3. Suppose that the functions $F(t, u, w)$ and $f(t, u, w)$ be continuous in the domain (1) and satisfy the inequalities (2) and (3). Then the solution (9) is stable for all $t \geq 0$. 
Raad N. Butris et al., International Journal of Information Systems and Computer Sciences, 9(1), January - February 2020,5 -10

Proof. Taking

$\|\mathrm{u}(\mathrm{t})-\overline{\mathrm{u}}(\mathrm{t})\| \leq\|\mathrm{f}(\mathrm{a})-\overline{\mathrm{f}}(\mathrm{a})\|+\mathrm{K}_{\alpha} \frac{\mathrm{h}^{\alpha}}{\alpha \Gamma(\alpha)}(\|\mathrm{u}(\mathrm{t})-\overline{\mathrm{u}}(\mathrm{t})\|+$

$$
\|\mathrm{w}(\mathrm{t})-\overline{\mathrm{w}}(\mathrm{t})\|)
$$

and

$\|\mathrm{w}(\mathrm{t})-\overline{\mathrm{w}}(\mathrm{t})\| \leq\|\mathrm{g}(\mathrm{a})-\overline{\mathrm{g}}(\mathrm{a})\|+\mathrm{L}_{\alpha} \frac{\mathrm{h}^{\alpha}}{\alpha \Gamma(\alpha)}(\|\mathrm{u}(\mathrm{t})-\overline{\mathrm{u}}(\mathrm{t})\|+$

$$
\|\mathrm{w}(\mathrm{t})-\overline{\mathrm{w}}(\mathrm{t})\|) \text {, }
$$

where

$$
\overline{\mathrm{u}}=\overline{\mathrm{f}}(\mathrm{a})+\frac{1}{\Gamma(\alpha)} \int_{\mathrm{a}}^{\mathrm{t}}(\mathrm{t}-\mathrm{s})^{\alpha-1} \mathrm{~F}(\mathrm{t}, \mathrm{s}, \overline{\mathrm{u}}(\mathrm{s}), \overline{\mathrm{w}}(\mathrm{s})) \mathrm{ds}
$$

and

$$
\overline{\mathrm{w}}=\overline{\mathrm{g}}(\mathrm{a})+\frac{1}{\Gamma(\alpha)} \int_{\mathrm{a}}^{\mathrm{b}}(\mathrm{t}-\mathrm{s})^{\alpha-1} \mathrm{H}(\mathrm{t}, \mathrm{s}, \mathrm{u}(\mathrm{s}), \mathrm{w}(\mathrm{s})) \mathrm{ds}
$$

Rewrite (24) and (25) in a vector form that is

$\left(\begin{array}{c}\|\mathrm{u}(\mathrm{t})-\overline{\mathrm{u}}(\mathrm{t})\| \\ \|\mathrm{w}(\mathrm{t})-\overline{\mathrm{w}}(\mathrm{t})\|\end{array}\right) \leq\left(\begin{array}{c}\|\mathrm{f}(\mathrm{a})-\overline{\mathrm{f}}(\mathrm{a})\| \\ \|\mathrm{g}(\mathrm{a})-\overline{\mathrm{g}}(\mathrm{a})\|\end{array}\right)+\omega\left(\begin{array}{c}\|\mathrm{u}(\mathrm{t})-\overline{\mathrm{u}}(\mathrm{t})\| \\ \|\mathrm{w}(\mathrm{t})-\overline{\mathrm{w}}(\mathrm{t})\|\end{array}\right)$

For

$$
\begin{aligned}
& \|\mathrm{f}(\mathrm{a})-\overline{\overline{\mathrm{f}}(\mathrm{a})}\| \leq \delta_{1},\|\mathrm{~g}(\mathrm{a})-\overline{\mathrm{g}}(\mathrm{a})\| \leq \delta_{2} \quad \text { then } \\
& \left(\begin{array}{c}
\|\mathrm{u}(\mathrm{t})-\overline{\mathrm{u}}(\mathrm{t})\| \\
\|\mathrm{w}(\mathrm{t})-\overline{\mathrm{w}}(\mathrm{t})\|
\end{array}\right) \leq\left(\begin{array}{c}
\delta_{1} \\
\delta_{2}
\end{array}\right)+\omega\left(\begin{array}{c}
\|\mathrm{u}(\mathrm{t})-\overline{\mathrm{u}}(\mathrm{t})\| \\
\|\mathrm{w}(\mathrm{t})-\overline{\mathrm{w}}(\mathrm{t})\|
\end{array}\right)
\end{aligned}
$$

By using the condition (5) we have:

$$
\left(\begin{array}{c}
\|\mathrm{u}(\mathrm{t})-\overline{\mathrm{u}}(\mathrm{t})\| \\
\|\mathrm{w}(\mathrm{t})-\overline{\mathrm{w}}(\mathrm{t})\|
\end{array}\right) \leq\left(\begin{array}{c}
\epsilon_{1} \\
\epsilon_{2}
\end{array}\right), \epsilon_{1}, \epsilon_{2} \geq 0
$$

Also, by using the definition of the stability, we find that $\left(\begin{array}{c}\bar{u}(t) \\ \bar{w}(t)\end{array}\right)$ is a stable solution for $\mathrm{t} \geq 0$ of $(\mathrm{V})$ and $(\mathrm{F})$. Similar results can be obtained for other class of fractional integral equations of Volterra-Fridlhom types. In particular, the fractional integral equations which has the form:

$$
\begin{aligned}
& \mathrm{u}(\mathrm{t})=\mathrm{f}(\mathrm{t})+\frac{1}{\Gamma(\alpha)} \int_{\mathrm{a}}^{\mathrm{t}}(\mathrm{t}-\mathrm{s})^{\alpha-1} \mathrm{~F}(\mathrm{t}, \mathrm{s}, \mathrm{u}(\mathrm{s}), \mathrm{w}(\mathrm{s})) \mathrm{ds} \\
& \text { and } \\
& \mathrm{w}(\mathrm{t})=\mathrm{g}(\mathrm{t})+\frac{1}{\Gamma(\beta)} \int_{\mathrm{a}}^{\mathrm{b}}(\mathrm{t}-\mathrm{s})^{\beta-1} \mathrm{G}(\mathrm{t}, \mathrm{s}, \mathrm{u}(\mathrm{s}), \mathrm{w}(\mathrm{s})) \mathrm{ds}
\end{aligned}
$$

The vector functions $\mathrm{f}(\mathrm{t}), \mathrm{g}(\mathrm{t})$ and $\mathrm{F}(\mathrm{t}, \mathrm{u}, \mathrm{w}), \mathrm{G}(\mathrm{t}, \mathrm{u}, \mathrm{w})$ are define and continuous on the domain:-

$\mathrm{D}=\left\{(\mathrm{t}, \mathrm{s}, \mathrm{u}, \mathrm{w}) ; \mathrm{t}, \mathrm{s} \in \mathrm{R}^{1},|t-s| \leq h, \mathrm{u} \in \mathrm{D}_{1}, \mathrm{w} \in \mathrm{D}_{2}, 0<\propto\right.$

$$
, \beta<1\}
$$

Suppose that the vector functions $\mathrm{F}(\mathrm{t}, \mathrm{u}, \mathrm{w})$ and $\mathrm{G}(\mathrm{t}, \mathrm{u}, \mathrm{w})$ satisfy the following inequalities:

$\|\mathrm{F}(\mathrm{t}, \mathrm{s}, \mathrm{u}, \mathrm{w})\| \leq \mathrm{M}_{\alpha}, \quad\|\mathrm{G}(\mathrm{t}, \mathrm{s}, \mathrm{u}, \mathrm{w})\| \leq \mathrm{N}_{\beta}$ $\left\|\mathrm{F}\left(\mathrm{t}, \mathrm{s}, \mathrm{u}_{1}, \mathrm{w}_{1}\right)-\mathrm{F}\left(\mathrm{t}, \mathrm{s}, \mathrm{u}_{2}, \mathrm{w}_{2}\right)\right\|$

$$
\leq \mathrm{K}_{\alpha}\left(\left\|\mathrm{u}_{1}-\mathrm{u}_{2}\right\|+\left\|\mathrm{w}_{1}-\mathrm{w}_{2}\right\|\right)
$$

$$
\begin{aligned}
\| \mathrm{G}\left(\mathrm{t}, \mathrm{s}, \mathrm{u}_{1}, \mathrm{w}_{1}\right)- & \mathrm{G}\left(\mathrm{t}, \mathrm{s}, \mathrm{u}_{2}, \mathrm{w}_{2}\right) \| \\
& \leq \mathrm{L}_{\beta}\left(\left\|\mathrm{u}_{1}-\mathrm{u}_{2}\right\|+\left\|\mathrm{w}_{1}-\mathrm{w}_{2}\right\|\right)
\end{aligned}
$$

For,all $\mathrm{t}, \mathrm{s} \in \mathrm{R}^{1}, \mathrm{u}, \mathrm{u}_{1}, \mathrm{u}_{2} \in \mathrm{D}_{1}, \mathrm{w}, \mathrm{w}_{1}, \mathrm{w}_{2} \in \mathrm{D}_{2}$, where $\mathrm{M}_{\alpha}, \mathrm{N}_{\beta}, \mathrm{K}_{\alpha}$ and $\mathrm{L}_{\beta}$ are positive constant We define non-empty sets as follows: -

$$
\left.\begin{array}{c}
\mathrm{D}_{\mathrm{F}}=\mathrm{D}_{1}-\frac{\mathrm{h}^{\alpha} \mathrm{M}_{\alpha}}{\alpha \Gamma(\alpha)}+q_{1}+\|\mathrm{f}(\mathrm{a})\| \\
\mathrm{D}_{\mathrm{G}}=\mathrm{D}_{2}-\frac{\mathrm{h}^{\beta} \mathrm{N}_{\beta}}{\beta \Gamma(\beta)}+q_{2}+\|\mathrm{g}(\mathrm{a})\|
\end{array}\right\}
$$

Where, $\quad q_{1}=\max _{\mathrm{t} \in[0, \mathrm{~h}]}|f(t)|$ and $q_{2}=\max _{\mathrm{t} \in[0, \mathrm{~h}]}|g(t)|$

Furthermore, we assume that the largest Eigen-value of the matrix

$\omega=\left(\begin{array}{ll}\mathrm{K}_{\alpha} \frac{\mathrm{h}^{\alpha}}{\alpha \Gamma(\alpha)} & \mathrm{K}_{\alpha} \frac{\mathrm{h}^{\alpha}}{\alpha \Gamma(\alpha)} \\ \mathrm{L}_{\beta} \frac{\mathrm{h}^{\beta}}{\beta \Gamma(\beta)} & \mathrm{L}_{\beta} \frac{\mathrm{h}^{\beta}}{\beta \Gamma(\beta)}\end{array}\right)$

less than one, i.e.

$$
\varphi_{\max }(\omega)=\mathrm{K}_{\alpha} \frac{\mathrm{h}^{\alpha}}{\alpha \Gamma(\alpha)}+\mathrm{L}_{\beta} \frac{\mathrm{h}^{\beta}}{\beta \Gamma(\beta)}<1
$$

Define a sequence of functions $\left\{\mathrm{u}_{\mathrm{m}}(\mathrm{t})\right\}_{\mathrm{m}=0}^{\infty},\left\{\mathrm{w}_{\mathrm{m}}(\mathrm{t})\right\}_{\mathrm{m}=0}^{\infty}$ by the following: -

$\mathrm{u}_{\mathrm{m}}(\mathrm{t})=\mathrm{f}(\mathrm{t})+\frac{1}{\Gamma(\alpha)} \int_{\mathrm{a}}^{\mathrm{t}}(\mathrm{t}-\mathrm{s})^{\alpha-1} \mathrm{~F}\left(\mathrm{t}, \mathrm{s}, \mathrm{u}_{\mathrm{m}-1}(\mathrm{~s}), \mathrm{w}_{\mathrm{m}-1}(\mathrm{~s})\right) \mathrm{ds}$

With $\quad \mathrm{u}_{0}(\mathrm{a})=\mathrm{f}(\mathrm{a})$

and

$\mathrm{w}_{\mathrm{m}}(\mathrm{t})=\mathrm{g}(\mathrm{t})+\frac{1}{\Gamma(\beta)} \int_{\mathrm{a}}^{\mathrm{b}}(\mathrm{t}-\mathrm{s})^{\beta-1} \mathrm{H}\left(\mathrm{t}, \mathrm{s}, \mathrm{u}_{\mathrm{m}-1}(\mathrm{~s}), \mathrm{w}_{\mathrm{m}-1}(\mathrm{~s})\right) \mathrm{ds}$

With $\quad \mathrm{w}_{0}(\mathrm{a})=\mathrm{g}(\mathrm{a})$

We can state and prove a similar theorem of a theorem 1 by using the condition (29).

\section{EXAMPLES}

Example (1): consider the following system of fractional integro-differential equations, for $\mathrm{t} \in \mathrm{I}=[1,5]$, $\alpha=0.1,0.9, \quad u(1)=1, w(1)=3$ Picard approximation solutions using MATLAB are show in figure (1):

$$
\begin{aligned}
u(t)=\frac{t^{3}+1}{\Gamma(\alpha+1)} & +\frac{1}{\Gamma(\alpha)} \int_{1}^{t}(t-s)^{\alpha-1}(-0.8 \mathrm{u}(\mathrm{s}) \\
& +\ln (\mathrm{w}(\mathrm{s})) d s
\end{aligned}
$$


Raad N. Butris et al., International Journal of Information Systems and Computer Sciences, 9(1), January - February 2020,5 -10

$w(t)=\frac{t^{2}}{\Gamma(\alpha+1)}+\frac{1}{\Gamma(\alpha)} \int_{1}^{5}(t-s)^{\alpha-1}(\ln (u(s)+w(s)) d s$

\begin{tabular}{|c|c|c|c|c|}
\hline & \multicolumn{2}{|c|}{$\alpha=0.1$} & \multicolumn{2}{c|}{$\alpha=0.9$} \\
\hline$t_{i}$ & $u\left(t_{i}\right)$ & $w\left(t_{i}\right)$ & $u\left(t_{i}\right)$ & $w\left(t_{i}\right)$ \\
\hline 1 & 3.1022 & 4.0511 & 3.0795 & 4.0397 \\
\hline 1.1 & 3.6995 & 6.776 & 3.4627 & 4.6507 \\
\hline 1.2 & 4.475 & 9.475 & 3.9213 & 5.2864 \\
\hline 1.3 & 5.0723 & 12.2748 & 4.4612 & 5.9755 \\
\hline 1.4 & 5.8366 & 15.0739 & 5.0878 & 6.7267 \\
\hline 1.5 & 6.5849 & 17.9617 & 5.8068 & 7.5465 \\
\hline
\end{tabular}
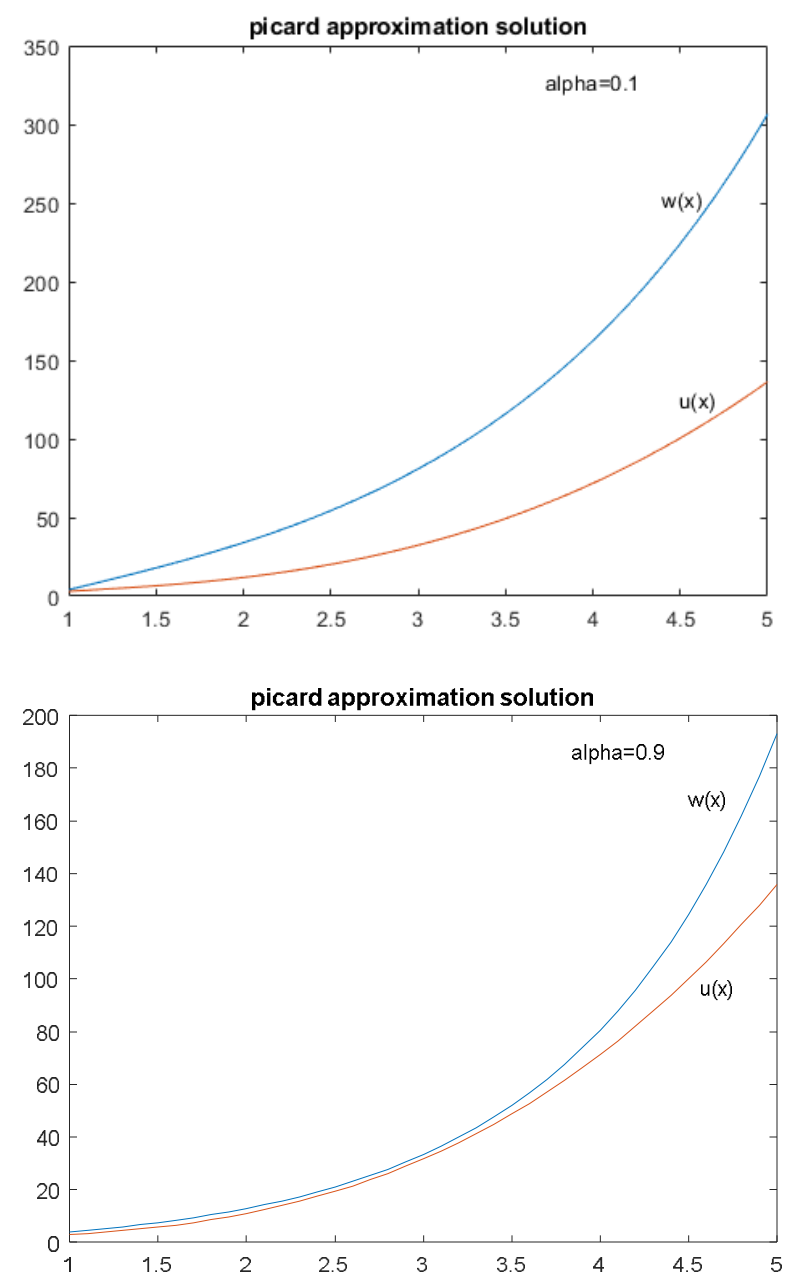

Figure (1)

Example (2): consider the following system of fractional integro-differential equations, for $\mathrm{t} \in \mathrm{I}=[1,5], \alpha=$ $0.01,0.8, u_{o}(1)=0, w_{0}(5)=1$ : numerical solution using MATLAB approximation method are show in figure (2):

$$
u(t)=\frac{0.99 e^{t-1}}{\Gamma(\alpha)}+\frac{1}{\Gamma(\alpha)} \int_{1}^{t}(t-s)^{\alpha-1} \frac{\ln (u(s)+2 w(s))}{2} d s
$$

$$
w(t)=\frac{-1.33 e^{t+1}}{\Gamma(\alpha)}+\frac{1}{\Gamma(\alpha)} \int_{1}^{5}(t-s)^{\alpha-1} \frac{\sin (5 \mathrm{u}(\mathrm{s})+w(s))}{3} d s
$$

\begin{tabular}{|c|c|c|c|c|}
\hline & \multicolumn{2}{|c|}{$\alpha=0.8$} & \multicolumn{2}{c|}{$\alpha=0.01$} \\
\hline$t_{i}$ & $u\left(t_{i}\right)$ & $w\left(t_{i}\right)$ & $u\left(t_{i}\right)$ & $w\left(t_{i}\right)$ \\
\hline 1 & 0.8503 & -7.4411 & 0.0099 & 0.9011 \\
\hline 1.1 & 0.9987 & -8.2811 & 0.3516 & 1.1664 \\
\hline 1.2 & 1.1476 & -9.2194 & 0.5363 & 0.9343 \\
\hline 1.3 & 1.3060 & -10.2649 & 0.4944 & 0.7012 \\
\hline 1.4 & 1.4762 & -11.4308 & 0.3975 & 0.8203 \\
\hline 1.5 & 1.6593 & -12.7312 & 0.4364 & 0.9213 \\
\hline
\end{tabular}
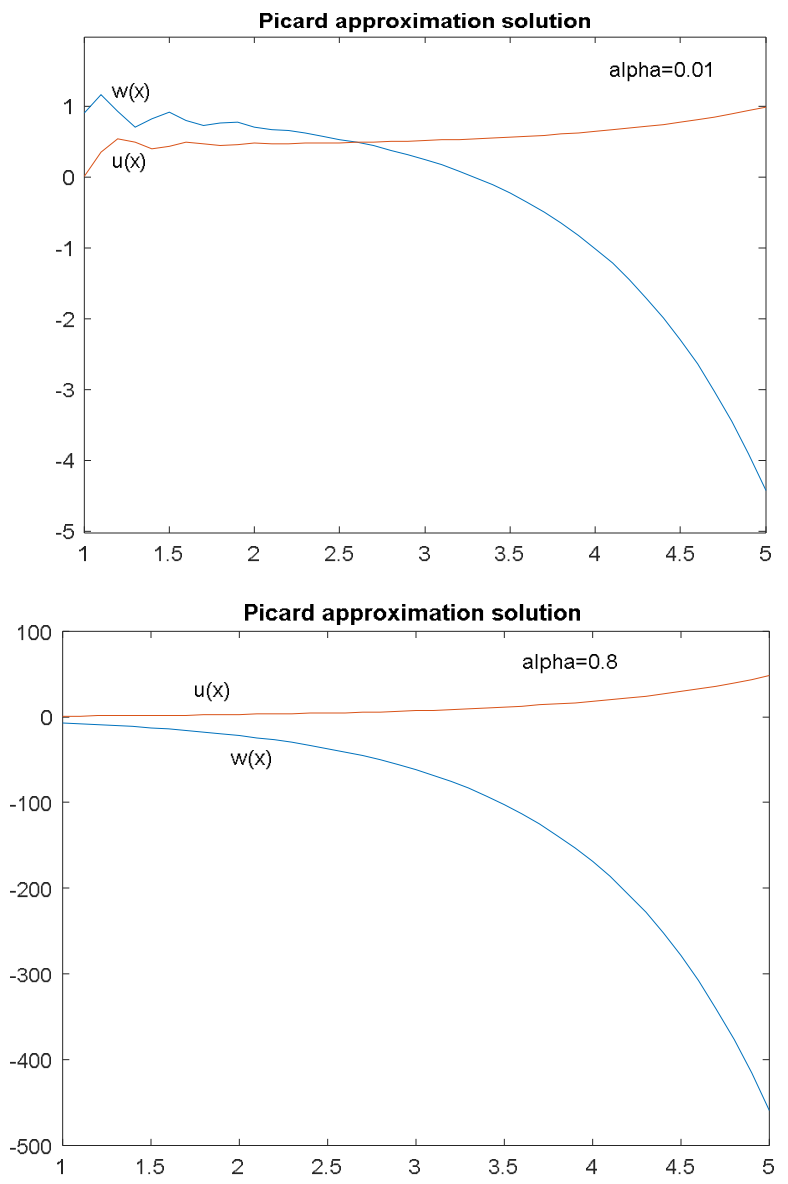

Figure (2)

\section{CONCLUSION}

In this work the existence uniqueness and stability solutions of new fractional integral equations of Volterra-Fridlhom types is studied by using Picard approximation method. Theorems on existence and uniqueness of a solutions are established under some necessary and sufficient conditions on compact spaces.

\section{REFERENCES}

[1] Butris, R. N. Solutions for the Volterra integral equations of the second kind, university of Mosul, Mosul, Iraq, (1984). 
[2]Butris, R. N., and Rafeq, A. Sh., Existence and Uniqueness Solution for Nonlinear Volterra Integral Equation, J. Duhok Univ. Vol. 14, No. 1, (Pure and Eng. Sciences), (2011). https://doi.org/10.1017/CBO9780511543234

[3] Brunner, H. Collocation methods for Volterra integral and related functional differential equations (Vol. 15). Cambridge University Press. (2004).

[4] Burton, T. A. Volterra integral and differential equations Vol. 202, Elsevier. (2005).

[5] Coddington, E. A. and Levinson, N., Theory of Ordinary Differential Equations, Mc Graw-Hill Book Company, New York, (1955).

[6] Grald, T., Topics in Real and Functional Analysis, American Mathematical society Rhcde Island,( 2010).

[7] Diethelm, K. An algorithm for the numerical solution of differential equations of fractional order, Electron. Trans. Number. Anal. 5 (1997), 1-6.

[8] Diethelm, K. and Walz, G., Numerical solution of fractional order differential equations by extrapolation, Numer. Algorithms 16 (1997), 231-253. https://doi.org/10.1023/A:1019147432240

[9] Glockle, W. G. and Nonnenmacher ,T. F., A fractional calculus approach to self-similar protein dynamics, Biophys. J. 68 (1995), 46-53.

[10] Guoqiang, H. and Ruifang, W., (2001), The extrapolation method for two-dimensional Volterra integral equations based on the asymptotic expansion of iterated galerkin solutions, Journal of Integral Equationsand Applications, Vol.13, No. 1, Spring. https://doi.org/10.1216/jiea/996986881

[11] Hendi, F. A. and Al-Hazm, Sh., (2010), The non-linear Volterra integral equation with weakly kernels and toeplitz matrix method, Vol. 3, No.2.

[12] Jaswon, M. A., \& Symm, G. T. Integral equation methods in potential theory and electrostatics Vol. 132, (1977).

[13] Maleknejad, K., \& Aghazadeh, N. Numerical solution of Volterra integral, (2005).

[14] Mattheij and Molenaar, Ordinary Differential Equations in Theory and Practice, Jhon Wiley \& Sons, New Yourk, (1996).

[15] Putetnka, T, V., The convergent method for nonlinear systems of differential equations with boundary integral conditions Nonlinear problems in the theory of differential equations. J. Akad, Nauk, Kive, Ukraine Vol.9 (1991).

[16] Plaat,O., Ordinary Differential Equations, Holden-Day, Inc.San Francisco, Cambridge,Londan, A msterdam, (1971).

[17] Rama, M.M., Ordinary Differential Equations Theory and Applications, United Kingdom, (1981).

[18] Rafeeg, A. sh., Periodic solutions for some classes of nonlinear of integro- differential equations Thesis, Iraq. Duhok, Univ. of Duhok, (2009).

[19] Samoilenko. A.M. and Ronto. N.I., A, numerical analytic methods for investigations of periodic solutions, Kiev, Ukraine, (1976)

[20] Struble, R. A., Non-Linear Differential Equations, Mc Graw- Hall Book Company Inc., New York,(1962).

[21] Tarang, M. Stability of the spline collocation method for Volterra integral equations, (2004).

https://doi.org/10.3846/13926292.2004.9637243
[22]Tricomi ,F,G.Integral Equations, Turin University, Turin, Italy, (1965). 